\title{
Bioinformatics, Genomics, and Proteomics Tools in Drug Design
}

\author{
Farid Shokry Ataya ${ }^{1,2, *}$ \\ ${ }^{1}$ Department of Biochemistry, College of Science, King Saud University, Saudi Arabia \\ ${ }^{2}$ Molecular Biology Department, Genetic Engineering Division, National Research Centre, Egypt
}

*Corresponding author: Farid Shokry Ataya, Department of Biochemistry, College of Science, King Saud University, Kingdom of Saudi Arabia, E-mail: fataya@ksu.edu.sa

Received: 15 Feb, 2019 | Accepted: 25 Mar, 2019 | Published: 29 Mar, 2019

Citation: Ataya FS (2019) Bioinformatics, Genomics, and Proteomics Tools in Drug Design. J Drug Res Dev 5(1): dx.doi.org/10.16966/24701009.147

Copyright: (C) 2019 Ataya FS. This is an open-access article distributed under the terms of the Creative Commons Attribution License, which permits unrestricted use, distribution, and reproduction in any medium, provided the original author and source are credited.

\begin{abstract}
Unraveling of human genome sequences had enabled the discovery of new drugs, studies of their effects, and determination of their main targets. Integrated genomics, proteomics, and bioinformatics have led to powerful new strategies for solving many biochemical issues and developing new techniques which give new biomedical products. Among the applications were analyses of DNA and genome annotation, predicting target structure, and understanding the genetics of disease. As a result, a new trend in research had evolved to illustrate the mechanism of drug action, predict of drug resistance, and discover biomarkers for many diseases. The discovery of new drugs is complex process. Drug design is based on 2 main strategies: ligand-based drug design and structure-based drug design. Studying protein structure and function was the key to develop drug in which modern techniques were used based on combinatorial approaches like proteomics, genomics, bioinformatics, molecular docking and mass spectrometry. To successfully design a new drug, it is important to identify the cell or tissue targeted by this drug. No absolute confirmation that the suspected drug will be effective in vivo. In this review, an overview on the combinatorial approaches of proteomics, genomics and bioinformatics that help understanding the process of drug design is discussed.
\end{abstract}

Keywords: Ligand-based drug design; Structure-based drug design; Target structure; Molecular docking; Mass spectrometry

\section{Introduction}

Drug design is the development of new medications that interact with biological targets. The word "drug" is derived from the primitive German word "dreug" which means "to dry". This word was used to describe the process of preparing medicinal products from herbs in dried powder form [1]. Most drugs are organic small molecules that can alter the function of a specific gene, protein, or receptor, which in turn affects their biochemical functions. In the past two decades, the science of designing drugs that are complementary in structure to or bind with their target has greatly advanced $[2,3]$. There are many limitations to successful drug discovery such as thermostability, metabolic half-life, side effects, and immunological reactions. Some of these factors are difficult to predict using rational drug design techniques [4] and many studies must be conducted in vitro, using cell lines, and in experimental animals before drugs are approved for human use [5]. Determining the genomes of many living organisms has advanced proteomics techniques and bioinformatics, facilitating drug design. Genomics is the study of the structure and function of the genetic information in an organism. The genome is the complete collection of genetic information of a living organism and can be passed from parents to their offspring. Proteomics is essentially concerned with studying protein structure, function, localization, and targeting. Most genomics studies initially examine genes and the translation to protein, whereas proteomics studies characterize functionally modified proteins. The development of bioinformatics tools has enabled researchers to design, compare, and predict the structure and function of genes. Bioinformatics tools are also used to analyze proteins and predict their 3D structures based on known model proteins $[6,7]$ and study the binding of ligands or drugs to specific binding sites [8].

The process of drug design and approval of new drugs for human use is complicated and requires combined knowledge from many fields. This leads to the production of novel biomedical products, understanding of biochemical pathways, and development of new methods for resolving medical conditions. The aim of this review was to provide insights into the role and recent advances of three important biotechnology research fields in drug design and discovery, including the relationship between genomics, proteomics, and bioinformatics and identification of drug targets and their applications in drug design, followed by a discussion of drug discovery.

\section{Drug Design and Discovery}

Drug discovery is a multi-process that involves genomics, proteomics, and bioinformatics studies, which lead to the discovery of a new drug entity with a novel mechanism of action. Proper drug design is a crucial step in the process of drug discovery, requiring great effort and up to 9 years research involving different disciplines and advanced lab techniques, as well as numerous clinical investigations. 
The evolution of drug design depends on many factors including identification of the increasing number of new drug targets (gene, protein, or receptor), accumulated data obtained from X-ray crystallographic studies revealing the three-dimensional structures of thousands of proteins (PDB database), advances in computing power of bioinformatics, and availability of tools for predicting protein-drug interactions [9].

\section{Types of drug design}

Two major strategies were used for drug design: ligand-based and structure-based techniques.

- Ligand-based drug design relies on knowledge of a known chemical (typically a drug, inhibitor, or cofactor) that binds to the target of interest and uses this compound as a model for drug to examine its binding to a known target [10].

- Structure-based drug design depends on the X-ray crystallographic structure of a known protein target or homology model of an unstudied protein compared to a known structure [7,11]. This type is divided into three methods [12]:

» Identification of a drug that fits into the binding pocket of a given receptor by searching large databases of $3 \mathrm{D}$ structures using fast approximate docking programs.

» De novo building up or design of new ligands within the constraints of the binding pocket. This can be conducted by assembling small pieces of chemical structure (atoms or molecular fragments) in a stepwise manner.

» Evaluating the binding of the studied drug to the binding cavity using a well-studied drug as a reference.

\section{Relations of Genomics, Proteimics and Bioinformatics to Drug Design}

The genome of bacteriophage lambda was the first genome sequenced, which opened a new era of research to obtain information related to open reading frames (sequences that are translated into protein). Subsequently, the genomes of many organisms were determined from microbial, viral, organelle and human sources (genome list https://www.ncbi.nlm.nih.gov/genome/browse/\#!/ overview/) [13]. Drugs are designed to bind a gene or protein. Thus, identifying the molecule targeted by a drug is important. After cloning and sequencing the gene of interest and predicting the corresponding amino acids, the sequence is compared using the Basic Local Alignment Search Tool (BLAST) and the relevant functional motifs of the known protein can be identified [14,15].

A thorough understanding of the structure and chemical properties of amino acid residues in protein targets or active groups is needed for predicting correct drug/target interactions. This process is facilitated by many bioinformatics programs that use "molecular dynamics, molecular mechanics" and other computational methods to calculate ligand-binding affinity and estimate intermolecular interactions between the designed drug and its target. These methods can predict the shape of a drug and any conformational changes in the targeted protein upon drug binding. Computerized semi-empirical methods, quantum chemistry methods, or density functional theory are often used to estimate the electrostatic potential, polarizability, hydrophobicity, ionic and non-ionic interactions, thermal stability, and other factors that may influence the aggregation of subunits and binding affinity between the ligand candidate and its target [16-18].

Correct design and effective use of computational methods will save enormous time and cost compared to the use of traditional methods for drug discovery. Once the bioinformatics methods for drug design are completed, the compound is synthesized and further examined. Although great advances in the field of computational biology have enormously accelerated drug design and discovery, much more effort is needed to develop new technologies and programs that accelerate drug discovery [17].

Proteomics is the science of examining protein expression levels, modification, stability, action, and degradation. It uses both traditional and modern techniques to explore protein structure, function, and turnover.

Proteins are encoded by genes and exert numerous functions. Thus, determining the link between genomics and proteomics is crucial [19].

Most drugs target proteins or enzymes by binding to a specific site in the active form of the protein to affect its function. Data obtained from genome analysis and transcription studies of specific genes are not sufficient to ensure high active protein yield. The expression "one gene, one protein" is not accurate, as it has been confirmed that the average number of protein isoforms per gene is one or two in bacteria, three in yeast, and three or more in humans because of the formation of protein isoforms produced by different forms of posttranslational modifications (phosphorylation, glycosylation, nitrosation, ethylation, and methylation) [20]. Bioinformatics analysis may reveal potential posttranslational modifications in certain proteins. Epigenetic factors may also affect protein expression and modifications. Thus, the human body may contain $>500,000$ modified proteins. Ligand binding sites can also be predicted using bioinformatics tools, starting from predicted amino acid residues of each protein.

Proteomics studies have broadened the perspective of protein research to examine the consequences of protein function in establishing the phenotype and interaction of a drug with its target. These studies have also revealed the pathophysiological basis of disease, validation of drug targets, action, toxicity, and side effects [20].

\section{Identification of Target Structure}

A drug target is a biomolecule involved in a specific metabolic pathway or modified in a specific disease and be targeted by successful drug [21]. The drug can be a small molecule designed to increase or decrease target function in a specific manner and hence modify certain biochemical pathways in the diseased organism. The target can be a receptor, protein, enzyme, or nucleic acid [22]. Many types of drugs may act as receptor antagonists, modulators, activators, or inhibitors of some enzymes or even effectors of membrane channels by opening or blocking the passage of important ions [23]. Some drugs are designed to be complementary to the target's binding site, while others block the target [24].

Genomics and proteomics research tools are employed to localize targets [25]. These methods are used to study the differences in protein expression in different tissues to distinguish between normal and diseased persons and enable the identification of specific biomarkers [26]. The study of proteins may involve a cell, tissue, or entire organism [27] and be conducted in situ, in vitro, or in vivo. In situ protein labeling with fluorescent dyes is a well-established technique for comparative proteomics. In vitro analysis is also useful and employs classical and modern techniques in protein analysis such as electrophoretic separation, isoelectric focusing, molecular weight determination, western blot analysis, two-dimensional gel electrophoresis, and mass spectrometry $[20,28,29]$. 


\section{Discovery of drug targets}

Many genome projects have mapped the DNA of human and other organisms. Advances in the field of proteomics and bioinformatics have revealed an ever-increasing number of proteins as potential drug targets. Using bioinformatics, a similar or closely related target and their binding sites in an unknown protein can be identified by sequence homology studies using a known protein structure. These unidentified targets are known as orphan receptors. The first step in drug discovery is identifying disease-associated targets. Most disease cases are associated with alterations in gene and/or protein expression and posttranslational modifications.

Once a drug target has been identified, its corresponding gene is cloned and expressed, and the expressed protein is purified to homogeneity for further analysis. In addition, the three-dimensional structure of the target may be determined.

Target specificity is a crucial factor in drug design and discovery, and can differ between different species and within tissues of the same species. The specificity of penicillin as an antibacterial agent is a good example of this effect in different species, as penicillin targets the enzyme necessary for bacterial cell wall synthesis, which is lacking in mammals. Tissue-specific drug targeting is another example; drugs targeting a specific receptor in an organ or tissue in the same species are identified. For example, the discovery of drugs targeting $\beta 2$ adrenergic receptors in the lung but not $\beta 1$ adrenergic receptors in the heart is challenging. This drug would enable treatment of the lung with minimal side effects in the heart and vice versa [22].

\section{Biochemical identification and characterization of the drug target}

Target identification and studies of drug-target interactions are multi-step processes that depend on modern proteomics techniques including purification, expression, flow cytometry, microarray, biochemical analysis, immunological techniques, X-ray crystallography, NMR, and MALDI-TOF. Techniques for testing a drug, target, or both should be simple, rapid, accurate, and relevant, particularly when evaluating large numbers of samples.

Production and purification of recombinant protein: Previously, a long time was required to isolate and purify sufficient quantities of target proteins for analysis. However, recent advances in molecular biology techniques have enabled researchers to produce large quantities of protein in a few hours through genetic engineering by incorporating the gene corresponding to the protein of interest into a fast-growing host under a powerful promoter. First, a pair of primers is designed by bioinformatics programs to amplify the gene corresponding to the protein via polymerase chain reaction. The amplified gene is sequenced, and the sequence is compared to those in a database using various bioinformatics tools. The gene is cloned into an expression vector using restriction enzymes under the control of a powerful promotor in a suitable host for producing sufficient quantities of recombinant protein. The recombinant protein can be purified easily by affinity chromatography using different affinity matrices [30,31]. For example, HIV protease is expressed and purified in Escherichia coli [32].

Profiling gene expression of protein target: Examining gene or protein expression profiles in disease and drug treatment using genomics and proteomics techniques offers a basis for detecting drug targets. In the past few years, researchers have identified protein targets for many diseases using genomic and proteomic approaches [33].
Microarrays or DNA chips are powerful tools for genome analysis. The genomes of hundreds of organisms are being sequenced, and the genomes of approximately 30 model organisms have been revealed. These genomic data are helpful for functional predictions of specific genes, particularly for studying the biological effect and potency of a designed drug targeting such genes. Data obtained can be used to screen race-specific genes, virulence genes, and conserved genes of pathogenic organisms, specific bacterial or viral enzyme genes, and bacterial membrane-translocation proteins [34]. Gene microarray can be used to evaluate the expression levels of certain genes in different patients or different tissues of the same patient and analyze gene function and regulation during differentiation periods and pathological change conditions [35].

Specific binding of cDNA or oligonucleotide sequences of interest with extracted nucleic acid (labeled with fluorescent dye) enables researchers to profile the gene expression patterns of tens of thousands of genes in a single experiment. The labeled isolated RNA binds quantitatively to its complementary sequences and the difference in fluorescence intensities between the test samples and control reflects the level of expression of the gene, which can be measured with a laser scanner [13].

Gene expression levels can be estimated by RT-PCR, in which a gene of interest in cDNA or RNA is amplified by PCR using specific primers and expression is monitored by the fluorescence emitted from Sybr Green dye. Expression of the test sample is compared with that of a steadily expressed gene known as a housekeeping gene. RT-PCR can be used to analyze the expression of many genes in a quantitative manner during treatment or in different tissues of the same organism $[13,36]$.

Two-dimensional electrophoresis (2DE): 2DE separates a mixture of proteins from different sources based on their isoelectric points and molecular weights. Using this technique, we can analyze proteomes of cell, tissue, and serum, and detect posttranslational modifications after gene expression, such as phosphorylation, glycosylation, hydroxylation, etc. [13].

MALDI-TOF and mass spectrometry (MS): Matrix-Assisted Laser Desorption/Ionization-Time of Flight (MALDI-TOF) is a modern technique that revolutionized research in the fields of peptides, proteins, nucleic acids, glyco-conjugates, and synthetic polymers.

In MALDI-TOF, expressed proteins are differentially digested with a protease to produce peptides and then these peptides are ionized at high sensitivity by a laser coupled and the ion mass to charge ratio is detected with a mass analyzer. The resulting peptides can be further characterized by tandem mass spectrometry (MS/MS) with a second MS analyzer that produces an MS/MS spectrum representing a series of ion fragments of the specific peptide. This technique is informative because it provides information about both peptide masses and the amino acid sequence [13].

\section{Assay of drug-target interactions}

Studies of drug-target interactions can be conducted in vitro or in vivo.

In vitro assay of drug-target interactions: In vitro assays are required to generate preliminary results which can be used to gain ethical approval for in vivo studies. In vitro studies are conducted using isolated and purified proteins, enzymes, cells, or tissues. These processes are advantageous because they are inexpensive, simple, noncontroversial, and can be automated. 
A target protein binding site can be tested by adding a specific drug that may be a substrate, cofactor, or inhibitor [37]; for enzymes, we can predict if this ligand binds to the substrate binding site to result in competitive inhibition, another site in the enzyme's backbone to result in non-competitive inhibition, or substrate resulting in uncompetitive inhibition.

In vivo assay of drug-target interactions: In vivo assays represent the next step. First, the drug is tested for its biological effect, toxicity, immunological reactions, etc., in experimental animals before being tested in human volunteers. One of the main limitations of this method is the genetic differences between experimental animals and humans in their responses to certain drugs. Scientists have overcome this problem by generating a series of transgenic animal models in which their genetic material is altered, and certain genes are replaced with human genes to produce the desired human target to be studied. Alternatively, mouse genes can be mutated to become susceptible to certain diseases. The most commonly used models are carcinogenic rats [22].

Identification of the binding site in the target molecule by NMR: After purification and characterization of the target, the next step is to identify the key residues in the binding site that determine the space and chemical environment around it. The binding site can be identified by X-ray crystallography [37] or NMR [22] as a concave pocket on the surface of a protein target that accommodates drug molecules through hydrophobic interactions, hydrogen bonding, among others, to drive drug binding $[38,39]$.

NMR can be used to detect whether a drug binds to its protein target by exposing it to radiation waves. This energy excites the nuclei of specific atoms such as hydrogen, carbon, or nitrogen. The relaxation time needed for each atom to release the excess energy to return to the ground state depends on the type of atom and environment and space around it, providing information about the structure of the ligand and how it binds to the target [22]. Many computational methods are used to determine the various components of ligands and target proteins. These methods predict the changes in polar and non-polar areas upon ligand binding and estimate the desolvation energy, number of rotatable bonds, configurational or strain energy, and number of hydrogen bonds formed [40].

\section{Drug Design by Molecular Docking}

Once the crystal structure of the target protein is known, molecular modeling software can be used to identify, predict, and design a ligand and study its binding to the target. Protein-ligand docking is a computer-based technique used to predict the position and orientation of a ligand in its binding site in the protein target. Moreover, by measuring the distance between different atoms in the binding site and ligand, it became simple to in silico predict the binding interaction and make changes in the design of the drug to improve binding.

\section{Molecular docking is a sequence series of computer modeling trials}

An example of molecular docking is shown for Acetyl Choline Esterase (AChE) as described by De Vita, et al. [41]:

- Downloading a crystal structure of a known AChE complexed with a natural ligand (such as donepezil) from the PDB website.

- Preparation of the PDB file for docking using the Dock Prep tool (UCSF Chimera 1.10.1) by adding hydrogen atoms, removing water molecules, and assigning partial charges (using the AMBER99 force field).
- Making the necessary docking calculations using Swiss Dock, which is freely available on the Swiss Institute of Bioinformatics Website (EADock DSS software) [42,43].

- Run docking (s) using the "Accurate" parameters option.

- Rank the Output Clusters after each run according to the Full Fitness (FF) scoring function (Swiss Dock algorithm). A cluster value of 0 means that the best FF score has been reached, while a greater negative FF score means that a more favorable binding mode with a better fit has been reached. Visualized data of the Swiss Dock by UCSF Chimera package.

- Repeat the docking experiment with a natural ligand (donepezil) to evaluate the ability of the software to correctly recognize the AChE-binding site.

However, not all targets can be crystalized after binding to the drug to study their active sites by X-ray or NMR. Hence, molecular modeling can be used to predict such interactions [44]. Further chemical experiments are needed to ensure the expected binding and biological activity of such drugs [22].

\section{Properties of chemical groups in ligand are essential for good design}

Recognizing certain functional groups is essential for properly understanding the mechanism of drug-target interactions. Not all functional groups participate in binding; for example, an alkyl group in a ligand can either bind to the target, help place the drug in the correct orientation in the protein's binding pocket, or protect the drug during its journey through the body.

It is known that the chemical structure of the drug is complementary to the binding region in the target. Thus, previous knowledge of the structure of the target or ligand using certain software tools and data from specialized databases can help predict the structure of the binding site of the target or ligand and modify the structure to alter their characteristics. For example, replacing a specific chemical group of a drug with another group may improve both the biological activity of the drug and its binding to the target, as well as reveal chemical nature of the binding site [22].

- Drugs containing alcohol or phenol, i.e. those with an $-\mathrm{OH}$ group, are typically involved in forming hydrogen bonds with a binding site containing $-\mathrm{OH},-\mathrm{NH}$, and $\mathrm{SH}$, on serine, glutamine, and cysteine, respectively.

- Drugs containing an aromatic ring or alkanes are typically involved in binding to tryptophan or phenyl alanine via hydrophobic interactions or van der Waal forces in the binding site.

- Drugs containing ketones and aldehydes form hydrogen bonds through a carbonyl group, which can act as a hydrogen acceptor from a hydrogen donor in the target binding site.

- Drugs containing an amine can act as both a hydrogen donor and acceptor depending the protonation state. Amines can form also strong ionic bonds with the carboxylate group of aspartate or glutamate.

- Drugs containing amide groups or in the form of amino acid derivatives bind to the target through hydrogen bonding.

- Drugs with a quaternary ammonium salt interact via ionic bonding with the carboxylate group of aspartic acid and glutamic acid. 
- Drugs with an -SH group bind to zinc ion and act as inhibitors of enzymes containing zinc cofactors, such as in the case of zinc metalloproteinases.

- Heterocyclic drugs contain one or more heteroatoms such as oxygen, nitrogen, or sulfur. Such compounds can interact with their targets through a variety of bonding forces such as van der Waal forces, hydrophobic interactions, and hydrogen and ionic bonding.

\section{Modification of drug structure}

Various chemical groups can be added to a drug structure to modify its biological or physical activity.

Optimization of the chemical structure of the drug to improve activity: Using molecular docking and bioinformatics programs, the structure of a well-known drug can be modified to overcome various limitations such as low activity, poor selectivity, side effects, or even difficulty in the synthesis of such compounds. Identifying drug analogs with improved properties depends on optimizing its interaction with the binding site in the target molecule. Many different strategies can be used, as follows:

- Variation in substituent's. This approach includes alkyl and aromatic group substitution. An example of alkyl substitution is replacing the methyl group in adrenaline with isopropyl to give isoprenaline. An example of aromatic substitution is a sulfonamide substituent at position 7 in the benzopyran structure, which increases antiarrhythmic drug activity.

- Extension of the structure. There are many methods for changing the length of a side chain to modify drug activity.

Optimization of drug for better physical properties: Variation in the structure of a drug may affect its access to the target by affecting its solubility, stability, and toxicity. The drug absorption can be improved by changing alkyl, acyl, $\mathrm{N}$-acetyl group, etc. to vary polarity. Drug stability and resistance to chemical and enzymatic degradation and decreasing its metabolism can be modified by introducing an ester group. Also the drug targeting to certain tissues such as by targeting tumor cells via a monoclonal antibody and targeting gastrointestinal tract infections can be optimized by fully ionized drug; e.g. sulfonamides. Finally, drug toxicity can be reduced by improving membrane permeability [22].

\section{Conclusion}

The unraveling of human genome sequence enabled many applications and ideas to discover new drugs, study their effect and determine their main target. Using bioinformatics and the knowledge in the fields of genomics and proteomics have led to build new powerful strategies to design new drugs. The accumulated knowledge in these fields helps in DNA and genome annotation, predicting protein structure, and understanding the genetics of disease. As a result, a new trend in pharmaceutical research has evolved to illustrate the mechanism of drug action, prediction of drug resistance, and discovery of biomarkers for many diseases.

\section{References}

1. Erhardt $P$ (2009) Drug Discovery. In: Hacker M, Messer WS, Bachmann KA (eds) Pharmacology: Principles and Practice. Academic Press, USA 475-560.

2. Misko TA, Liu YT, Harris ME, Oleinick NL, Pink J, et al. (2019) Structure-guided design of anti-cancer ribonucleotide reductase inhibitors. J Enzyme Inhib Med Chem 34: 438-450.
3. Mishra P, Kumar A, Panda G (2019) Anti-cholinesterase hybrids as multi-target-directed ligands against Alzheimer's disease (19982018). Bio org Med Chem.

4. Waring MJ, Arrowsmith J, Leach AR, Leeson PD, Mandrell S, et al. (2015) An analysis of the attrition of drug candidates from four major pharmaceutical companies. Nat Rev Drug Discov 14: 475-486.

5. Vuorinen A, Bellion P, Beilstein P (2017) Applicability of in silico genotoxicity models on food and feed ingredients. Regul Toxicol Pharmacol 90: 277-288.

6. Chronopoulou EG, Papageorgiou AC, Ataya F, Nianiou-Obeidat I, Madesis P, et al. (2018) Expanding the plant GSTome through directed evolution: DNA shuffling for the generation of new synthetic enzymes with engineered catalytic and binding properties. Front Plant Sci 9: 1737.

7. Marinou M, Platis D, Ataya FS, Chronopoulou E, Vlachakis D, et al. (2018) Structure-Based Design and Application of a Nucleotide Coenzyme Mimetic Ligand: Application to the Affinity Purification of Nucleotide Dependent Enzymes. J Chromatogr A 1535: 88-100.

8. Hartmann T, Middendorf M, Bernt M (2018) Genome rearrangement analysis: cut and join genome rearrangements and gene cluster preserving approaches. Methods Mol Biol 1704: 261-289.

9. Jones LH, Neubert H (2017) Clinical chemoproteomics-Opportunities and obstacles. Sci Transl Med.

10. Leelananda SP, Lindert S (2016) Computational methods in drug discovery. Beilstein J Org Chem 12: 2694-2718.

11. Śledź P, Caflisch A (2018) Protein structure-based drug design: from docking to molecular dynamics. Curr Opin Struct Biol 48: 93-102.

12. Klebe G (2000) Recent developments in structure-based drug design. J Mol Med 78: 269-281.

13. Wang Y, Chiu JF, He QY (2009) Genomics and Proteomics in Drug Design and Discovery. In: Hacker M, Messer WS, Bachmann KA (eds) Pharmacology: Principles and Practice. Academic Press, USA 561573.

14. Singh G, Singh V (2018) Functional elucidation of hypothetical proteins for their indispensable roles toward drug designing targets from Helicobacter pylori strain HPAG1. J Biomol Struct Dyn 36: 906918.

15. Perperopoulou F, Ataya FS, Fouad D, Malik A, Saeed HM, et al. (2016) Biochemical characterization of the detoxifying enzyme glutathione transferase P1-1 from the Camel Camelus Dromedarius. Cell Biochemistry and Biophysics 74: 459-472.

16. deAzevedo Jr WF, Dias R (2008) Computational methods for calculation of ligand-binding affinity. Curr Drug Targets 9: 10311039.

17. Lewis RA (2011) The Development of Molecular Modelling Programs: The Use and Limitations of Physical Models. In: Livingstone DJ, Davis AM (eds) Drug Design Strategies: Quantitative Approaches. Royal Society of Chemistry,UK 88-107.

18. Malik A, Khan JM, Alamery SF, Fouad D, Labrou NE, et al. (2018) Monomeric Camelus dromedarius GSTM1 at low pH is structurally more thermostable than its native dimeric form. PLoS One 13: e0205274.

19. Okerberg ES, Wu J, Zhang B, Samii B, Blackford K, et al. (2005) Highresolution functional proteomics by active-site peptide profiling. Proc Natl Acad Sci USA 102: 4996-5001. 
20. Zhang HM, Nan ZR, Hui GQ, Liu XH, Sun Y (2014) Application of genomics and proteomics in drug target discovery. Genet Mol Res 13: $198-204$

21. Vizovišek M, Vidmar R, Fonović M, Turk B (2016) Current trends and challenges in proteomic identification of protease substrates. Biochimie 122: 77-87.

22. Patrick GL (2013) Drug discovery: finding a lead. In: Patrick GL (eds) An introduction to medicinal chemistry. $5^{\text {th }}$ edition, Oxford Press, UK 189.

23. Imming P, Sinning C, Meyer A (2006) Drugs, their targets and the nature and number of drug targets. Nat Rev Drug Discov 5: 821-834.

24. Anderson AC (2003) The process of structure-based drug design. Chem Biol 10: 787-797.

25. Frantzi M, Latosinska A, Mischak H (2019) Proteomics in Drug Development: The Dawn of a New Era? Proteomics Clin Appl 5: e1800087.

26. Hanash S (2003) Disease proteomics. Nature 422: 226-232.

27. Dear JW, Leelahavanichkul A, Aponte A, Hu X, Constant SL, et al. (2007) Liver proteomics for therapeutic drug discovery: inhibition of the cyclophilin receptor CD147 attenuates sepsis-induced acute renal failure. Crit Care Med 35: 2319-2328.

28. Ünlü M, Morgan ME, Minden JS (1997) Difference gel electrophoresis: A single gel method for detecting changes in protein extracts. Electrophoresis 18: 2071-2077.

29. Saeed H, Shalaby M, Embaby A, Ismael M, Pathan A, et al. (2015) The Arabian camel Camelus dromedarius heat shock protein 90a: cDNA cloning, characterization and expression. Int J Biol Macromol 81: 195-204.

30. Ataya FS, Witte CP, Galván A, Igeño MI, Fernández E (2003) Mcp1 Encodes the Molybdenum Cofactor Carrier Protein in Chlamydomonas reinhardtii and Participates in Protection, Binding, and Storage Functions of the Cofactor. J Biol Chemistry 278: 1088510890.

31. Saeed H, Ali H, Soudan H, Embaby A, El-Sharkawy A, et al. (2018) Molecular cloning, structural modeling and production of recombinant Aspergillus terreus L. asparaginase in Escherichia coli. Int J Biol Macromol 106: 1041-1051.
32. Azarnezhad A, Sharifi Z, Seyedabadi R, Hosseini A, Johari B, et al. (2016) Cloning and expression of soluble recombinant HIV-1 CRF35 Protease-HP thioredoxin fusion protein. Avicenna J Med Biotechnol 8: $175-181$

33. Ahn NG, Wang AH (2008) Proteomics and genomics: perspectives on drug and target discovery. Curr Opin Chem Biol 12: 1-3.

34. Zhang YD (2009) Bioinformatics. $2^{\text {nd }}$ edition. Science Press, Beijing.

35. Ferrer-Alcón $M$, Arteta $D$, Guerrero MJ, Fernandez-Orth D, Simón $L$, et al. (2009) The use of gene array technology and proteomics in the search of new targets of diseases for therapeutics. Toxicol Lett 186: 45-51.

36. Ataya FS (2012) Cloning, Phylogenetic Analysis and 3D Modeling of a Putative Lysosomal Acid Lipase from the Camel, Camelus dromedaries. Molecules 17: 10399-10413.

37. Fischer K, Llamas A, Tejada-Jimenez M, Schrader N, Kuper J, et al. (2006) Function and Structure of the Molybdenum Cofactor Carrier Protein from Chlamydomonas reinhardtii. J Biol Chem 28: 3018630194.

38. Cheng RKY, Abela R, Hennig M (2017) X-ray free electron laser: opportunities for drug discovery. Essays Biochem 61: 529-542.

39. Nitsche C, Otting G (2017) NMR studies of ligand binding. Curr Opin Struct Biol 48: 16-22.

40. Gramatica P (2011) Modeling Chemicals in the Environment. In: Livingstone DJ, Davis AM (eds) Drug Design Strategies: Quantitative Approaches. Royal Society of Chemistry 466.

41. De Vita D, Pandolfi F, Ornano L, Feroci M, Chiarotto I, et al. (2016) New $\mathrm{N}, \mathrm{N}$-dimethylcarbamate inhibitors of acetylcholinesterase: design synthesis and biological evaluation. J Enzyme Inhib Med Chem 31: 106-113.

42. Zoete V, Cuendet MA, Grosdidier A, Michielin O (2011) SwissParam: a fast force field generation tool for small organic molecules. Comput Chem 32: 2359-2368.

43. Grosdidier A, Zoete V, Michielin O (2011) SwissDock, a protein-small molecule docking web service based on EADock DSS. Nucleic Acids Res 39: W270-W277.

44. Saeed $\mathrm{H}$, Soudan $\mathrm{H}$, El-Sharkawy A, Farag A, Embaby A, et al. (2018) Expression and Functional Characterization of Pseudomonas aeruginosa Recombinant L.Asparaginase. Protein J 37: 461-471. 